Acta Crystallographica Section E

Structure Reports

Online

ISSN 1600-5368

\section{Daniel E. Lynch ${ }^{\mathrm{a}}$ and Ian McClenaghan $^{\mathbf{b}}$ *}

${ }^{a}$ School of Science and the Environment, Coventry University, Coventry CV1 5FB, England, and ${ }^{\mathbf{b}}$ Key Organics Ltd, Highfield Industrial Estate, Camelford, Cornwall PL32 9QZ, England

Correspondence e-mail: apx106@coventry.ac.uk

\section{Key indicators}

Single-crystal X-ray study

$T=120 \mathrm{~K}$

Mean $\sigma(\mathrm{C}-\mathrm{C})=0.003 \AA$

$R$ factor $=0.046$

$w R$ factor $=0.122$

Data-to-parameter ratio $=17.6$

For details of how these key indicators were automatically derived from the article, see http://journals.iucr.org/e.
(C) 2005 International Union of Crystallography Printed in Great Britain - all rights reserved

\title{
Diethyl 2-aminothiazole-4,5-dicarboxylate hemihydrate
}

The structure of the title compound, $\mathrm{C}_{9} \mathrm{H}_{12} \mathrm{~N}_{2} \mathrm{O}_{4} \mathrm{~S} \cdot 0.5 \mathrm{H}_{2} \mathrm{O}$, comprises two thiazole molecules and one water molecule in the asymmetric unit. The two thiazoles associate via $\mathrm{N}-$ $\mathrm{H} \cdots \mathrm{N}$ hydrogen-bonding interactions to form $R_{2}^{2}(8)$ graph-set dimers, while the other $\mathrm{NH}$ groups and water molecules construct a hydrogen-bonding network that involves three of the four ethyl ester carbonyl $\mathrm{O}$ atoms.

\section{Comment}

The title compound (I) was prepared as part of an ongoing investigation into the synthesis and structural properties of 2aminothiazole derivatives.<smiles>CCOC(=O)c1nc(N)sc1C(=O)OCC</smiles>

(I)

As a part of that study, we have been examining the hydrogen-bonding networks of 2-aminothiazoles with ethyl ester substituents at the 5-position. The addition of two hydrogen-bond acceptors to the existing hydrogen-bond acceptors in 2-aminothiazole has led to some interesting

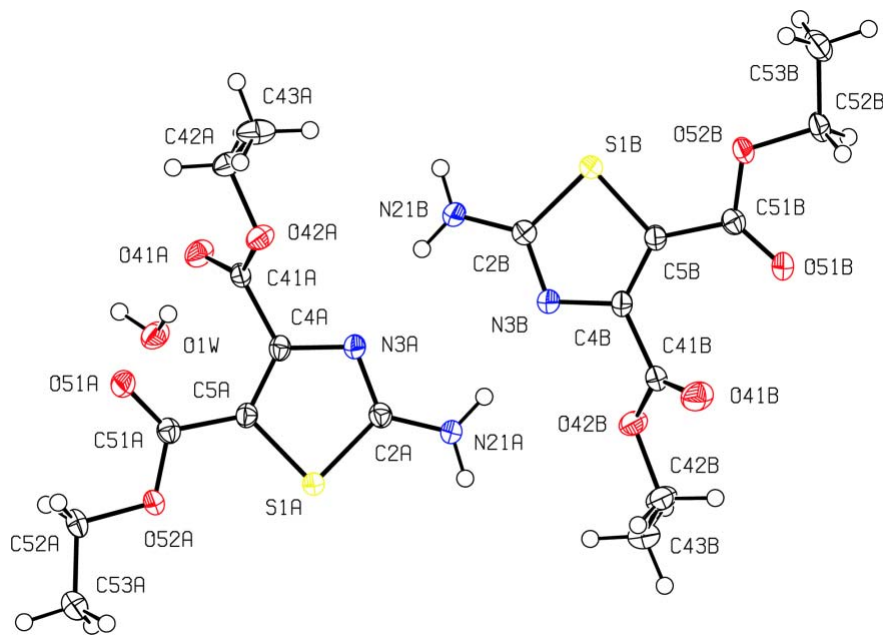

Figure 1

The structure of the asymmetric unit of (I). Displacement ellipsoids are drawn at the $50 \%$ probability level and $\mathrm{H}$ atoms are drawn as spheres of arbitrary radii.
Received 21 February 2005 Accepted 25 February 2005 Online 4 March 2005 


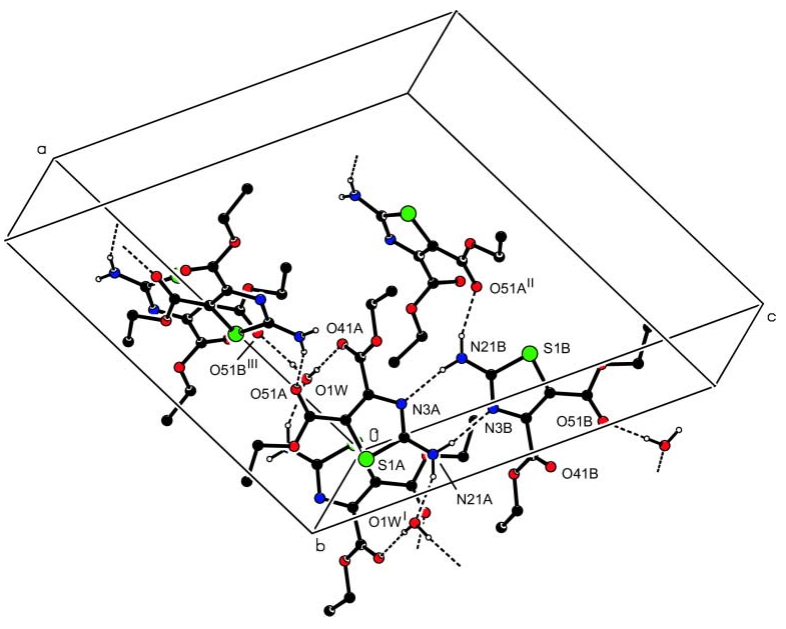

Figure 2

Partial packing diagram for (I). Hydrogen-bonding interactions are shown as dotted lines. $\mathrm{H}$ atoms not involved in the hydrogen-bonding network have been omitted for clarity. [Symmetry codes: (i) $-x,-y,-z$; (ii) $-x+\frac{1}{2}, y-\frac{1}{2},-z+\frac{1}{2}$; (iii) $x+\frac{1}{2},-y-\frac{1}{2}, z-\frac{1}{2}$.]

packing arrangements. For the three previous structures that contain a primary 2-amino group, viz. ethyl 2-amino-4-phenyl1,3-thiazole-5-carboxylate (Lynch \& McClenaghan, 2000), ethyl 2-amino-4-tert-butyl-1,3-thiazole-5-carboxylate (Lynch \& McClenaghan, 2004) and ethyl 2-amino-4-isopropyl-1,3thiazole-5-carboxylate (Kennedy et al., 2004), all molecules pack with one $\mathrm{NH}$ group interacting with an adjacent thiazole $\mathrm{N}$ atom, to form an $R_{2}^{2}(8)$ graph-set (Etter, 1990) dimer, while the other $\mathrm{NH}$ group associates with the ethyl ester carbonyl $\mathrm{O}$ atom. In the other four known thiazoles with ethyl ester substituents at the 5-position and substituted 2-amino $\mathrm{N}$ atoms, two have tertiary amino groups, viz. ethyl 2-(5ethoxycarbonyl-2-(pyrrol-1yl)thiazol-4yl-2-iminoethanoate (Brickute et al., 2002) and ethyl 4-phenyl-2-(pyrrolyl)-1,3thiazole-5-carboxylate (Lynch \& McClenaghan, 2002b), and the other two both contain competing carbonyl $\mathrm{O}$ atoms with which the single $\mathrm{NH}$ groups associate, viz. ethyl 2-[N(t-butoxycarbonyl)-L-alanylamino]-4-methyl-1,3-thiazole-5carboxylate (Singh et al., 2000) and ethyl 4-tert-butyl-2-(3phenylureido)-1,3-thiazole-5-carboxylate (Lynch \& McClenaghan, 2002a). All five structures containing at least one $\mathrm{NH}$ group show that an amino group prefers to interact with a carbonyl $\mathrm{O}$ atom, whether from the ester group or from another group in the molecule. With this in mind we decided to examine a 2-aminothiazole analogue with two ethyl ester substituents to see which substituted position would be preferred by the $\mathrm{NH}$ groups in the hydrogen-bonding network.

The asymmetric unit of (I) comprises two thiazole molecules; these are connected in a three-dimensional hydrogenbonding network. The addition of the second ethyl ester and thus the increase in hydrogen-bond acceptor atoms leads to the incorporation of a water molecule to satisfy the hydrogenbonding requirements of the packing network (Desiraju, 1991). Similarly to the three previous structures where the 2 amino group was unsubstituted, the two thiazole molecules in
(I) associate via $\mathrm{N}-\mathrm{H} \cdots \mathrm{N}$ interactions and form $R_{2}^{2}(8)$ graphset dimers (Fig. 1). The hydrogen-bonding geometry is listed in Table 1. The second NH group on each thiazole molecule individually associates with different hydrogen-bond acceptor sites; on molecule $A$, the second NH group hydrogen-bonds to the water $\mathrm{O}$ atom, while the second $\mathrm{NH}$ group on molecule $B$ hydrogen-bonds to the 5-substituted ester carbonyl $\mathrm{O}$ atom, on an adjacent molecule $\mathrm{A}$. In turn, the water $\mathrm{H}$ atoms are donors to the 4-substituted ester carbonyl $\mathrm{O}$ atom on an adjacent molecule $A$ and the equivalent 5-substituted $\mathrm{O}$ atom to molecule $B$; thus three of the four ester carbonyl $\mathrm{O}$ atoms are involved in the hydrogen-bonding network (Fig. 2). Separation distances of $\mathrm{O} 41 B$ (the remaining carbonyl $\mathrm{O}$ atom) of $3.208(2) \AA$ to $\mathrm{C} 52 A\left(-\frac{1}{2}+x, \frac{1}{2}-y, \frac{1}{2}+z\right)$ and 3.365 (2) $\AA$ to $\mathrm{C} 43 B\left(-\frac{1}{2}-x, \frac{1}{2}+y, \frac{1}{2}-z\right)$ indicate $\mathrm{C}-\mathrm{H} \cdots \mathrm{O}$ interactions.

\section{Experimental}

The title compound was obtained from Key Organics Ltd and crystals were grown from an ethanol solution.

\section{Crystal data}

$\mathrm{C}_{9} \mathrm{H}_{12} \mathrm{~N}_{2} \mathrm{O}_{4} \mathrm{~S} \cdot 0.5 \mathrm{H}_{2} \mathrm{O}$

$M_{r}=253.28$

Monoclinic, $P 2_{1} / n$

$a=16.7835(4) \AA$

$b=8.4580(2) \AA$

$c=18.0373(5) \AA$

$\beta=111.653(1)^{\circ}$

$V=2379.8(1) \AA^{3}$

$Z=8$

$$
\begin{aligned}
& D_{x}=1.414 \mathrm{Mg} \mathrm{m}^{-3} \\
& \text { Mo } K \alpha \text { radiation } \\
& \text { Cell parameters from } 5676 \\
& \quad \text { reflections } \\
& \theta=2.9-27.5^{\circ} \\
& \mu=0.28 \mathrm{~mm}^{-1} \\
& T=120(2) \mathrm{K} \\
& \text { Prism, colourless } \\
& 0.18 \times 0.15 \times 0.07 \mathrm{~mm}
\end{aligned}
$$

\section{Data collection}

Nonius KappaCCD diffractometer $\varphi$ and $\omega$ scans

Absorption correction: multi-scan (SADABS; Sheldrick, 2003)

$T_{\min }=0.952, T_{\max }=0.981$

28267 measured reflections

5447 independent reflections

\section{Refinement}

Refinement on $F^{2}$

$R\left[F^{2}>2 \sigma\left(F^{2}\right)\right]=0.046$

$w R\left(F^{2}\right)=0.122$

$S=1.09$

5447 reflections

\begin{tabular}{|c|c|c|c|c|}
\hline$D-\mathrm{H} \cdots A$ & $D-\mathrm{H}$ & $\mathrm{H} \cdots A$ & $D \cdots A$ & $D-\mathrm{H} \cdots A$ \\
\hline $\mathrm{N} 21 \mathrm{~A}-\mathrm{H} 21 \mathrm{~A} \cdots \mathrm{N} 3 \mathrm{~B}$ & 0.88 & 2.13 & $2.979(2)$ & 161 \\
\hline $\mathrm{N} 21 \mathrm{~A}-\mathrm{H} 22 \mathrm{~A} \cdots \mathrm{O} 1 \mathrm{~W}^{\mathrm{i}}$ & 0.88 & 1.97 & $2.805(2)$ & 158 \\
\hline $\mathrm{N} 21 \mathrm{~B}-\mathrm{H} 21 \mathrm{~B} \cdots \mathrm{N} 3 \mathrm{~A}$ & 0.88 & 2.16 & $3.005(2)$ & 160 \\
\hline $\mathrm{N} 21 \mathrm{~B}-\mathrm{H} 22 \mathrm{~B} \cdots \mathrm{O} 51 \mathrm{~A}^{\mathrm{ii}}$ & 0.88 & 2.05 & $2.890(2)$ & 160 \\
\hline $\mathrm{O} 1 \mathrm{~W}-\mathrm{H} 1 \mathrm{~W} \cdots \mathrm{O} 41 \mathrm{~A}$ & $0.83(3)$ & $2.02(3)$ & $2.853(2)$ & $175(2)$ \\
\hline $\mathrm{O} 1 \mathrm{~W}-\mathrm{H} 2 \mathrm{~W} \cdots \mathrm{O} 51 \mathrm{~B}^{\mathrm{iii}}$ & $0.84(3)$ & $1.97(3)$ & $2.808(2)$ & $175(2)$ \\
\hline
\end{tabular}

309 parameters

$\mathrm{H}$ atoms treated by a mixture of independent and constrained refinement

$$
\begin{aligned}
& 4260 \text { reflections with } I>2 \sigma(I) \\
& R_{\text {int }}=0.051 \\
& \theta_{\max }=27.5^{\circ} \\
& h=-21 \rightarrow 21 \\
& k=-10 \rightarrow 10 \\
& l=-23 \rightarrow 21
\end{aligned}
$$

$$
\begin{aligned}
& w=1 /\left[\sigma^{2}\left(F_{\mathrm{o}}{ }^{2}\right)+(0.0594 P)^{2}\right. \\
& +0.8191 P] \\
& \text { where } P=\left(F_{\mathrm{o}}{ }^{2}+2 F_{\mathrm{c}}{ }^{2}\right) / 3 \\
& (\Delta / \sigma)_{\max }<0.001 \\
& \Delta \rho_{\max }=0.56 \mathrm{e}^{-3} \\
& \Delta \rho_{\min }=-0.66 \mathrm{e}^{-3}
\end{aligned}
$$

Table 1

Hydrogen-bond geometry $\left(\AA{ }^{\circ}\right)$.

Symmetry codes: (i) $-x,-y,-z$; (ii) $-x+\frac{1}{2}, y-\frac{1}{2},-z+\frac{1}{2}$; (iii) $x+\frac{1}{2},-y-\frac{1}{2}, z-\frac{1}{2}$. 


\section{organic papers}

All $\mathrm{H}$ atoms, except the two water $\mathrm{H}$ atoms, were included in the refinement at calculated positions, in the riding-model approximation, with $\mathrm{C}-\mathrm{H}$ distances of $0.98\left(\mathrm{CH}_{3} \mathrm{H}\right.$ atoms $)$ and $0.99 \AA\left(\mathrm{CH}_{2} \mathrm{H}\right.$ atoms) and an $\mathrm{N}-\mathrm{H}$ distance of $0.88 \AA$. The two water $\mathrm{H}$ atoms were located in Fourier syntheses and positional parameters were refined. The isotropic displacement parameters for all $\mathrm{H}$ atoms were set equal to $1.25 U_{\text {eq }}$ of the carrier atom.

Data collection: COLLECT (Hooft, 1998); cell refinement: DENZO (Otwinowski \& Minor, 1997) and COLLECT; data reduction: DENZO and COLLECT; program(s) used to solve structure: SHELXS97 (Sheldrick, 1997); program(s) used to refine structure: SHELXL97 (Sheldrick, 1997); molecular graphics: PLATON97 (Spek, 2003); software used to prepare material for publication: SHELXL97.

The authors thank the EPSRC National Crystallography Service (Southampton, England).

\section{References}

Brickute, D., Slok, F. A., Romming, C. \& Sackus, A. (2002). J. Chem. Soc. Perkin Trans. 1, 652-656.

Desiraju, G. R. (1991). J. Chem. Soc. Chem. Commun. pp. 426-428.

Etter, M. C. (1990). Acc. Chem. Res. 23, 120-126.

Hooft, R. W. W. (1998). COLLECT. Nonius BV, Delft, The Netherlands.

Kennedy, A. R., Khalaf, A. I., Suckling, C. J. \& Waigh, R. D. (2004). Acta Cryst. E60, o1188-o1190.

Lynch, D. E. \& McClenaghan, I. (2000). Acta Cryst. C56, e586.

Lynch, D. E. \& McClenaghan, I. (2002a). Acta Cryst. E58, o733-o734.

Lynch, D. E. \& McClenaghan, I. (2002b). Acta Cryst. E58, o745-o746.

Lynch, D. E. \& McClenaghan, I. (2004). Acta Cryst. C60, o592-o594.

Otwinowski, Z. \& Minor, W. (1997). Methods in Enzymology. Vol. 276, Macromolecular Crystallography, Part A, edited by C. W. Carter Jr \& R. M. Sweet, pp. 307-326. New York: Academic Press.

Sheldrick, G. M. (1997). SHELXS97 and SHELXL97. University of Göttingen, Germany.

Sheldrick, G. M. (2003). SADABS. Version 2.10. Bruker AXS Inc., Madison, Wisconsin, USA.

Singh, U. P., Thomas, M., Seshadri, T. P. \& Bhattacharya, S. (2000). Acta Cryst. C56, 1482-1483.

Spek, A. L. (2003). J. Appl. Cryst. 367-13. 


\section{supporting information}

Acta Cryst. (2005). E61, o824-o826 [https://doi.org/10.1107/S1600536805006197]

\section{Diethyl 2-aminothiazole-4,5-dicarboxylate hemihydrate}

\section{Daniel E. Lynch and lan McClenaghan}

Diethyl 2-aminothiazole-4,5-dicarboxylate hemihydrate

Crystal data

$\mathrm{C}_{9} \mathrm{H}_{12} \mathrm{~N}_{2} \mathrm{O}_{4} \mathrm{~S} \cdot 0.5 \mathrm{H}_{2} \mathrm{O}$

$M_{r}=253.28$

Monoclinic, $P 2_{1} / n$

Hall symbol: -P 2 yn

$a=16.7835(4) \AA$

$b=8.4580(2) \AA$

$c=18.0373(5) \AA$

$\beta=111.653(1)^{\circ}$

$V=2379.80(10) \AA^{3}$

$Z=8$

Data collection

Nonius KappaCCD diffractometer

Radiation source: Bruker Nonius FR591 rotating anode

$10 \mathrm{~cm}$ confocal mirrors monochromator

Detector resolution: 9.091 pixels $\mathrm{mm}^{-1}$

$\varphi$ and $\omega$ scans

Absorption correction: multi-scan

(SADABS; Sheldrick, 2003)

\section{Refinement}

Refinement on $F^{2}$

Least-squares matrix: full

$R\left[F^{2}>2 \sigma\left(F^{2}\right)\right]=0.046$

$w R\left(F^{2}\right)=0.122$

$S=1.09$

5447 reflections

309 parameters

0 restraints

Primary atom site location: structure-invariant direct methods

Secondary atom site location: difference Fourier map
$F(000)=1064$

$D_{\mathrm{x}}=1.414 \mathrm{Mg} \mathrm{m}^{-3}$

Melting point $=377-378 \mathrm{~K}$

Mo $K \alpha$ radiation, $\lambda=0.71073 \AA$

Cell parameters from 5676 reflections

$\theta=2.9-27.5^{\circ}$

$\mu=0.28 \mathrm{~mm}^{-1}$

$T=120 \mathrm{~K}$

Prism, colourless

$0.18 \times 0.15 \times 0.07 \mathrm{~mm}$

$T_{\min }=0.952, T_{\max }=0.981$

28267 measured reflections

5447 independent reflections

4260 reflections with $I>2 \sigma(I)$

$R_{\text {int }}=0.051$

$\theta_{\max }=27.5^{\circ}, \theta_{\min }=3.2^{\circ}$

$h=-21 \rightarrow 21$

$k=-10 \rightarrow 10$

$l=-23 \rightarrow 21$

Hydrogen site location: inferred from neighbouring sites

$\mathrm{H}$ atoms treated by a mixture of independent and constrained refinement

$w=1 /\left[\sigma^{2}\left(F_{\mathrm{o}}^{2}\right)+(0.0594 P)^{2}+0.8191 P\right]$ where $P=\left(F_{\mathrm{o}}{ }^{2}+2 F_{\mathrm{c}}{ }^{2}\right) / 3$

$(\Delta / \sigma)_{\max }<0.001$

$\Delta \rho_{\max }=0.56 \mathrm{e} \AA^{-3}$

$\Delta \rho_{\min }=-0.66$ e $\AA^{-3}$

Extinction correction: SHELXL97, $\mathrm{Fc}^{*}=\mathrm{kFc}\left[1+0.001 \mathrm{xFc}^{2} \lambda^{3} / \sin (2 \theta)\right]^{-1 / 4}$

Extinction coefficient: 0.0145 (11) 
Special details

Experimental. The minimum and maximum absorption values stated above are those calculated in SHELXL97 from the given crystal dimensions. The ratio of minimum to maximum apparent transmission was determined experimentally as 0.847918 .

Fractional atomic coordinates and isotropic or equivalent isotropic displacement parameters $\left(\AA^{2}\right)$

\begin{tabular}{|c|c|c|c|c|}
\hline & $x$ & $y$ & $z$ & $U_{\text {iso }} * / U_{\text {eq }}$ \\
\hline S1A & $0.00585(3)$ & $0.19269(5)$ & $0.04174(3)$ & $0.01969(14)$ \\
\hline $\mathrm{C} 2 \mathrm{~A}$ & $0.00612(11)$ & $0.0765(2)$ & $0.12202(11)$ & $0.0200(4)$ \\
\hline $\mathrm{N} 21 \mathrm{~A}$ & $-0.06484(10)$ & $0.0536(2)$ & $0.13670(10)$ & $0.0251(4)$ \\
\hline $\mathrm{H} 21 \mathrm{~A}$ & -0.0635 & -0.0053 & 0.1774 & $0.031^{*}$ \\
\hline $\mathrm{H} 22 \mathrm{~A}$ & -0.1133 & 0.0973 & 0.1058 & $0.031^{*}$ \\
\hline N3A & $0.08201(9)$ & $0.01515(19)$ & $0.16560(9)$ & $0.0202(3)$ \\
\hline $\mathrm{C} 4 \mathrm{~A}$ & $0.14150(11)$ & $0.0590(2)$ & $0.13455(11)$ & $0.0185(4)$ \\
\hline $\mathrm{C} 41 \mathrm{~A}$ & $0.23107(11)$ & $-0.0028(2)$ & $0.17509(11)$ & $0.0187(4)$ \\
\hline O41A & $0.26555(8)$ & $-0.09470(17)$ & $0.14581(8)$ & $0.0274(3)$ \\
\hline $\mathrm{O} 42 \mathrm{~A}$ & $0.26563(8)$ & $0.05404(16)$ & $0.24851(8)$ & $0.0242(3)$ \\
\hline $\mathrm{C} 42 \mathrm{~A}$ & $0.35207(12)$ & $-0.0022(2)$ & $0.29540(12)$ & $0.0261(4)$ \\
\hline $\mathrm{H} 41 \mathrm{~A}$ & 0.3921 & 0.0256 & 0.2686 & $0.033^{*}$ \\
\hline $\mathrm{H} 42 \mathrm{~A}$ & 0.3521 & -0.1186 & 0.3015 & $0.033^{*}$ \\
\hline $\mathrm{C} 43 \mathrm{~A}$ & $0.37871(14)$ & $0.0766(3)$ & $0.37519(12)$ & $0.0341(5)$ \\
\hline $\mathrm{H} 43 \mathrm{~A}$ & 0.3802 & 0.1914 & 0.3684 & $0.043^{*}$ \\
\hline $\mathrm{H} 44 \mathrm{~A}$ & 0.4358 & 0.0393 & 0.4091 & $0.043^{*}$ \\
\hline $\mathrm{H} 45 \mathrm{~A}$ & 0.3375 & 0.0508 & 0.4003 & $0.043^{*}$ \\
\hline $\mathrm{C} 5 \mathrm{~A}$ & $0.11454(11)$ & $0.1528(2)$ & $0.06849(11)$ & $0.0189(4)$ \\
\hline $\mathrm{C} 51 \mathrm{~A}$ & $0.16686(12)$ & $0.2219(2)$ & $0.02763(11)$ & $0.0201(4)$ \\
\hline O51A & $0.24464(8)$ & $0.21272(17)$ & $0.05187(8)$ & $0.0262(3)$ \\
\hline O52A & $0.11926(8)$ & $0.29819(16)$ & -0.03851 & $0.0236(3)$ \\
\hline $\mathrm{C} 52 \mathrm{~A}$ & $0.16613(13)$ & $0.3716(3)$ & $-0.08358(13)$ & $0.0312(5)$ \\
\hline $\mathrm{H} 51 \mathrm{~A}$ & 0.1917 & 0.2895 & -0.1071 & $0.039^{*}$ \\
\hline $\mathrm{H} 52 \mathrm{~A}$ & 0.2127 & 0.4396 & -0.0483 & $0.039^{*}$ \\
\hline $\mathrm{C} 53 \mathrm{~A}$ & $0.10264(14)$ & $0.4683(3)$ & $-0.14800(13)$ & $0.0350(5)$ \\
\hline $\mathrm{H} 53 \mathrm{~A}$ & 0.0579 & 0.3991 & -0.1833 & $0.044^{*}$ \\
\hline H54A & 0.1319 & 0.5223 & -0.1789 & $0.044^{*}$ \\
\hline $\mathrm{H} 55 \mathrm{~A}$ & 0.0766 & 0.5469 & -0.1240 & $0.044^{*}$ \\
\hline S1B & $0.05291(3)$ & $-0.21687(6)$ & $0.43465(3)$ & $0.02324(14)$ \\
\hline $\mathrm{C} 2 \mathrm{~B}$ & $0.04552(12)$ & $-0.1559(2)$ & $0.34028(11)$ & $0.0234(4)$ \\
\hline $\mathrm{N} 21 \mathrm{~B}$ & $0.11009(10)$ & $-0.1726(2)$ & $0.31459(10)$ & $0.0310(4)$ \\
\hline $\mathrm{H} 21 \mathrm{~B}$ & 0.1041 & -0.1408 & 0.2664 & $0.039^{*}$ \\
\hline $\mathrm{H} 22 \mathrm{~B}$ & 0.1587 & -0.2154 & 0.3459 & $0.039^{*}$ \\
\hline N3B & $-0.02927(9)$ & -0.09242 (19) & $0.29602(9)$ & $0.0220(4)$ \\
\hline $\mathrm{C} 4 \mathrm{~B}$ & -0.08278 & $-0.0887(2)$ & 0.33755 (11) & $0.0196(4)$ \\
\hline $\mathrm{C} 5 \mathrm{~B}$ & $-0.05159(11)$ & $-0.1509(2)$ & $0.41288(11)$ & $0.0204(4)$ \\
\hline $\mathrm{C} 41 \mathrm{~B}$ & -0.16709 (11) & $-0.0072(2)$ & $0.29871(11)$ & $0.0213(4)$ \\
\hline O41B & $-0.19714(9)$ & $0.08482(18)$ & $0.33210(9)$ & $0.0331(4)$ \\
\hline O42B & $-0.20234(8)$ & $-0.04339(17)$ & $0.22165(8)$ & $0.0266(3)$ \\
\hline $\mathrm{C} 42 \mathrm{~B}$ & $-0.28320(12)$ & $0.0370(3)$ & $0.17790(12)$ & $0.0312(5)$ \\
\hline
\end{tabular}




$\begin{array}{lllll}\text { H41B } & -0.2726 & 0.1501 & 0.1707 & 0.039^{*} \\ \text { H42B } & -0.3221 & 0.0298 & 0.2078 & 0.039^{*} \\ \text { C43B } & -0.32269(13) & -0.0421(3) & 0.09868(12) & 0.0300(5) \\ \text { H43B } & -0.2853 & -0.0285 & 0.0683 & 0.037^{*} \\ \text { H44B } & -0.3788 & 0.0055 & 0.0692 & 0.037^{*} \\ \text { H45B } & -0.3299 & -0.1550 & 0.1065 & 0.037^{*} \\ \text { C51B } & -0.09509(12) & -0.1756(2) & 0.46898(11) & 0.0208(4) \\ \text { O51B } & -0.17060(8) & -0.15335(17) & 0.45389(8) & 0.0265(3) \\ \text { O52B } & -0.04057(8) & -0.23094(17) & 0.53874(8) & 0.0249(3) \\ \text { C52B } & -0.07804(13) & -0.2693(3) & 0.59773(12) & 0.0283(5) \\ \text { H51B } & -0.1233 & -0.3504 & 0.5764 & 0.035^{*} \\ \text { H52B } & -0.1039 & -0.1738 & 0.6114 & 0.035^{*} \\ \text { C53B } & -0.00694(14) & -0.3311(3) & 0.67031(13) & 0.0339(5) \\ \text { H53B } & 0.0398 & -0.2537 & 0.6879 & 0.042^{*} \\ \text { H54B } & 0.0145 & -0.4310 & 0.6572 & 0.042^{*} \\ \text { H55B } & -0.0287 & -0.3490 & 0.7131 & 0.042^{*} \\ \text { O1W } & 0.19277(9) & -0.21340(19) & -0.01321(9) & 0.0293(3) \\ \text { H1W } & 0.2123(15) & -0.173(3) & 0.0322(16) & 0.037^{*} \\ \text { H2W } & 0.2337(16) & -0.258(3) & -0.0215(15) & 0.037^{*}\end{array}$

Atomic displacement parameters $\left(\AA^{2}\right)$

\begin{tabular}{lllllll}
\hline & $U^{11}$ & $U^{22}$ & $U^{33}$ & $U^{12}$ & $U^{13}$ & $U^{23}$ \\
\hline S1A & $0.0172(2)$ & $0.0240(3)$ & $0.0185(2)$ & $0.00079(18)$ & $0.00726(18)$ & $0.00329(18)$ \\
C2A & $0.0211(9)$ & $0.0218(10)$ & $0.0183(9)$ & $-0.0003(7)$ & $0.0087(7)$ & $-0.0005(7)$ \\
N21A & $0.0179(8)$ & $0.0353(10)$ & $0.0243(9)$ & $0.0045(7)$ & $0.0103(7)$ & $0.0095(7)$ \\
N3A & $0.0186(7)$ & $0.0252(9)$ & $0.0187(8)$ & $0.0014(6)$ & $0.0089(6)$ & $0.0010(6)$ \\
C4A & $0.0188(8)$ & $0.0207(10)$ & $0.0179(9)$ & $-0.0014(7)$ & $0.0090(7)$ & $-0.0029(7)$ \\
C41A & $0.0181(8)$ & $0.0223(10)$ & $0.0174(9)$ & $-0.0022(7)$ & $0.0083(7)$ & $0.0018(7)$ \\
O41A & $0.0239(7)$ & $0.0358(8)$ & $0.0219(7)$ & $0.0064(6)$ & $0.0079(6)$ & $-0.0050(6)$ \\
O42A & $0.0199(6)$ & $0.0314(8)$ & $0.0193(7)$ & $0.0054(6)$ & $0.0049(5)$ & $-0.0042(6)$ \\
C42A & $0.0203(9)$ & $0.0299(11)$ & $0.0237(10)$ & $0.0042(8)$ & $0.0030(8)$ & $-0.0006(8)$ \\
C43A & $0.0345(11)$ & $0.0324(12)$ & $0.0253(11)$ & $0.0083(9)$ & $-0.0008(9)$ & $-0.0029(9)$ \\
C5A & $0.0170(8)$ & $0.0232(10)$ & $0.0173(9)$ & $-0.0013(7)$ & $0.0074(7)$ & $-0.0015(7)$ \\
C51A & $0.0212(9)$ & $0.0213(10)$ & $0.0183(9)$ & $-0.0024(7)$ & $0.0079(7)$ & $-0.0017(7)$ \\
O51A & $0.0181(7)$ & $0.0358(8)$ & $0.0237(7)$ & $-0.0039(6)$ & $0.0067(6)$ & $0.0023(6)$ \\
O52A & $0.0205(6)$ & $0.0314(8)$ & $0.0212(7)$ & $-0.0002(6)$ & $0.0103(5)$ & $0.0070(6)$ \\
C52A & $0.0294(10)$ & $0.0373(12)$ & $0.0337(12)$ & $0.0032(9)$ & $0.0196(9)$ & $0.0148(10)$ \\
C53A & $0.0362(12)$ & $0.0425(14)$ & $0.0319(12)$ & $0.0077(10)$ & $0.0190(10)$ & $0.0130(10)$ \\
S1B & $0.0187(2)$ & $0.0320(3)$ & $0.0195(2)$ & $0.00271(19)$ & $0.00761(19)$ & $0.00632(19)$ \\
C2B & $0.0214(9)$ & $0.0281(11)$ & $0.0211(10)$ & $0.0017(8)$ & $0.0084(8)$ & $0.0026(8)$ \\
N21B & $0.0208(8)$ & $0.0507(12)$ & $0.0245(9)$ & $0.0112(8)$ & $0.0120(7)$ & $0.0125(8)$ \\
N3B & $0.0194(8)$ & $0.0279(9)$ & $0.0203(8)$ & $0.0029(6)$ & $0.0092(6)$ & $0.0036(7)$ \\
C4B & $0.0195(9)$ & $0.0211(10)$ & $0.0211(9)$ & $-0.0007(7)$ & $0.0111(7)$ & $0.0001(7)$ \\
C5B & $0.0193(9)$ & $0.0240(10)$ & $0.0197(9)$ & $-0.0009(7)$ & $0.0092(7)$ & $0.0008(8)$ \\
C41B & $0.0204(9)$ & $0.0247(10)$ & $0.0207(10)$ & $-0.0008(8)$ & $0.0100(8)$ & $0.0036(8)$ \\
O41B & $0.0336(8)$ & $0.0369(9)$ & $0.0288(8)$ & $0.0103(7)$ & $0.0117(6)$ & $-0.0024(7)$ \\
O42B & $0.0217(7)$ & $0.0352(8)$ & $0.0214(7)$ & $0.0082(6)$ & $0.0060(6)$ & $-0.0005(6)$ \\
& & & & & &
\end{tabular}




\begin{tabular}{lllllll} 
C42B & $0.0227(10)$ & $0.0418(13)$ & $0.0264(11)$ & $0.0117(9)$ & $0.0058(8)$ & $0.0028(9)$ \\
C43B & $0.0268(10)$ & $0.0293(11)$ & $0.0293(11)$ & $0.0032(8)$ & $0.0052(9)$ & $0.0019(9)$ \\
C51B & $0.0226(9)$ & $0.0199(10)$ & $0.0206(9)$ & $-0.0026(7)$ & $0.0089(8)$ & $-0.0002(7)$ \\
O51B & $0.0218(7)$ & $0.0342(8)$ & $0.0271(8)$ & $-0.0002(6)$ & $0.0132(6)$ & $0.0048(6)$ \\
O52B & $0.0219(7)$ & $0.0367(8)$ & $0.0183(7)$ & $-0.0014(6)$ & $0.0099(6)$ & $0.0055(6)$ \\
C52B & $0.0285(10)$ & $0.0392(12)$ & $0.0224(10)$ & $-0.0019(9)$ & $0.0155(9)$ & $0.0050(9)$ \\
C53B & $0.0367(12)$ & $0.0424(13)$ & $0.0243(11)$ & $-0.0056(10)$ & $0.0133(9)$ & $0.0072(10)$ \\
O1W & $0.0224(7)$ & $0.0430(10)$ & $0.0219(7)$ & $0.0070(6)$ & $0.0075(6)$ & $-0.0025(7)$ \\
\hline
\end{tabular}

Geometric parameters $\left(\AA,{ }^{\circ}\right)$

\begin{tabular}{|c|c|c|c|}
\hline $\mathrm{S} 1 \mathrm{~A}-\mathrm{C} 5 \mathrm{~A}$ & $1.7392(18)$ & $\mathrm{S} 1 \mathrm{~B}-\mathrm{C} 5 \mathrm{~B}$ & $1.7418(18)$ \\
\hline $\mathrm{S} 1 \mathrm{~A}-\mathrm{C} 2 \mathrm{~A}$ & 1.7485 (19) & $\mathrm{C} 2 \mathrm{~B}-\mathrm{N} 3 \mathrm{~B}$ & $1.327(2)$ \\
\hline $\mathrm{C} 2 \mathrm{~A}-\mathrm{N} 21 \mathrm{~A}$ & $1.326(2)$ & $\mathrm{C} 2 \mathrm{~B}-\mathrm{N} 21 \mathrm{~B}$ & $1.334(2)$ \\
\hline $\mathrm{C} 2 \mathrm{~A}-\mathrm{N} 3 \mathrm{~A}$ & $1.331(2)$ & $\mathrm{N} 21 \mathrm{~B}-\mathrm{H} 21 \mathrm{~B}$ & 0.88 \\
\hline $\mathrm{N} 21 \mathrm{~A}-\mathrm{H} 21 \mathrm{~A}$ & 0.88 & $\mathrm{~N} 21 \mathrm{~B}-\mathrm{H} 22 \mathrm{~B}$ & 0.88 \\
\hline $\mathrm{N} 21 \mathrm{~A}-\mathrm{H} 22 \mathrm{~A}$ & 0.88 & $\mathrm{~N} 3 \mathrm{~B}-\mathrm{C} 4 \mathrm{~B}$ & $1.366(2)$ \\
\hline $\mathrm{N} 3 \mathrm{~A}-\mathrm{C} 4 \mathrm{~A}$ & $1.364(2)$ & $\mathrm{C} 4 \mathrm{~B}-\mathrm{C} 5 \mathrm{~B}$ & $1.368(3)$ \\
\hline $\mathrm{C} 4 \mathrm{~A}-\mathrm{C} 5 \mathrm{~A}$ & $1.363(3)$ & $\mathrm{C} 4 \mathrm{~B}-\mathrm{C} 41 \mathrm{~B}$ & $1.496(3)$ \\
\hline $\mathrm{C} 4 \mathrm{~A}-\mathrm{C} 41 \mathrm{~A}$ & $1.502(2)$ & $\mathrm{C} 5 \mathrm{~B}-\mathrm{C} 51 \mathrm{~B}$ & $1.465(3)$ \\
\hline $\mathrm{C} 41 \mathrm{~A}-\mathrm{O} 41 \mathrm{~A}$ & $1.201(2)$ & $\mathrm{C} 41 \mathrm{~B}-\mathrm{O} 41 \mathrm{~B}$ & $1.203(2)$ \\
\hline $\mathrm{C} 41 \mathrm{~A}-\mathrm{O} 42 \mathrm{~A}$ & $1.325(2)$ & $\mathrm{C} 41 \mathrm{~B}-\mathrm{O} 42 \mathrm{~B}$ & $1.330(2)$ \\
\hline $\mathrm{O} 42 \mathrm{~A}-\mathrm{C} 42 \mathrm{~A}$ & $1.463(2)$ & $\mathrm{O} 42 \mathrm{~B}-\mathrm{C} 42 \mathrm{~B}$ & $1.462(2)$ \\
\hline $\mathrm{C} 42 \mathrm{~A}-\mathrm{C} 43 \mathrm{~A}$ & $1.497(3)$ & $\mathrm{C} 42 \mathrm{~B}-\mathrm{C} 43 \mathrm{~B}$ & $1.493(3)$ \\
\hline $\mathrm{C} 42 \mathrm{~A}-\mathrm{H} 41 \mathrm{~A}$ & 0.99 & $\mathrm{C} 42 \mathrm{~B}-\mathrm{H} 41 \mathrm{~B}$ & 0.99 \\
\hline $\mathrm{C} 42 \mathrm{~A}-\mathrm{H} 42 \mathrm{~A}$ & 0.99 & $\mathrm{C} 42 \mathrm{~B}-\mathrm{H} 42 \mathrm{~B}$ & 0.99 \\
\hline $\mathrm{C} 43 \mathrm{~A}-\mathrm{H} 43 \mathrm{~A}$ & 0.98 & $\mathrm{C} 43 \mathrm{~B}-\mathrm{H} 43 \mathrm{~B}$ & 0.98 \\
\hline $\mathrm{C} 43 \mathrm{~A}-\mathrm{H} 44 \mathrm{~A}$ & 0.98 & $\mathrm{C} 43 \mathrm{~B}-\mathrm{H} 44 \mathrm{~B}$ & 0.98 \\
\hline $\mathrm{C} 43 \mathrm{~A}-\mathrm{H} 45 \mathrm{~A}$ & 0.98 & $\mathrm{C} 43 \mathrm{~B}-\mathrm{H} 45 \mathrm{~B}$ & 0.98 \\
\hline $\mathrm{C} 5 \mathrm{~A}-\mathrm{C} 51 \mathrm{~A}$ & $1.460(2)$ & $\mathrm{C} 51 \mathrm{~B}-\mathrm{O} 51 \mathrm{~B}$ & $1.209(2)$ \\
\hline $\mathrm{C} 51 \mathrm{~A}-\mathrm{O} 51 \mathrm{~A}$ & $1.217(2)$ & $\mathrm{C} 51 \mathrm{~B}-\mathrm{O} 52 \mathrm{~B}$ & $1.338(2)$ \\
\hline $\mathrm{C} 51 \mathrm{~A}-\mathrm{O} 52 \mathrm{~A}$ & $1.332(2)$ & $\mathrm{O} 52 \mathrm{~B}-\mathrm{C} 52 \mathrm{~B}$ & $1.458(2)$ \\
\hline $\mathrm{O} 52 \mathrm{~A}-\mathrm{C} 52 \mathrm{~A}$ & $1.462(2)$ & $\mathrm{C} 52 \mathrm{~B}-\mathrm{C} 53 \mathrm{~B}$ & $1.503(3)$ \\
\hline $\mathrm{C} 52 \mathrm{~A}-\mathrm{C} 53 \mathrm{~A}$ & $1.497(3)$ & $\mathrm{C} 52 \mathrm{~B}-\mathrm{H} 51 \mathrm{~B}$ & 0.99 \\
\hline $\mathrm{C} 52 \mathrm{~A}-\mathrm{H} 51 \mathrm{~A}$ & 0.99 & $\mathrm{C} 52 \mathrm{~B}-\mathrm{H} 52 \mathrm{~B}$ & 0.99 \\
\hline $\mathrm{C} 52 \mathrm{~A}-\mathrm{H} 52 \mathrm{~A}$ & 0.99 & $\mathrm{C} 53 \mathrm{~B}-\mathrm{H} 53 \mathrm{~B}$ & 0.98 \\
\hline $\mathrm{C} 53 \mathrm{~A}-\mathrm{H} 53 \mathrm{~A}$ & 0.98 & $\mathrm{C} 53 \mathrm{~B}-\mathrm{H} 54 \mathrm{~B}$ & 0.98 \\
\hline $\mathrm{C} 53 \mathrm{~A}-\mathrm{H} 54 \mathrm{~A}$ & 0.98 & $\mathrm{C} 53 \mathrm{~B}-\mathrm{H} 55 \mathrm{~B}$ & 0.98 \\
\hline $\mathrm{C} 53 \mathrm{~A}-\mathrm{H} 55 \mathrm{~A}$ & 0.98 & $\mathrm{O} 1 \mathrm{~W}-\mathrm{H} 1 \mathrm{~W}$ & $0.83(3)$ \\
\hline $\mathrm{S} 1 \mathrm{~B}-\mathrm{C} 2 \mathrm{~B}$ & $1.739(2)$ & $\mathrm{O} 1 \mathrm{~W}-\mathrm{H} 2 \mathrm{~W}$ & $0.84(3)$ \\
\hline $\mathrm{C} 5 \mathrm{~A}-\mathrm{S} 1 \mathrm{~A}-\mathrm{C} 2 \mathrm{~A}$ & $88.82(9)$ & $\mathrm{N} 3 \mathrm{~B}-\mathrm{C} 2 \mathrm{~B}-\mathrm{N} 21 \mathrm{~B}$ & $122.89(17)$ \\
\hline $\mathrm{N} 21 \mathrm{~A}-\mathrm{C} 2 \mathrm{~A}-\mathrm{N} 3 \mathrm{~A}$ & $124.00(17)$ & $\mathrm{N} 3 \mathrm{~B}-\mathrm{C} 2 \mathrm{~B}-\mathrm{S} 1 \mathrm{~B}$ & $114.77(14)$ \\
\hline $\mathrm{N} 21 \mathrm{~A}-\mathrm{C} 2 \mathrm{~A}-\mathrm{S} 1 \mathrm{~A}$ & $121.29(14)$ & $\mathrm{N} 21 \mathrm{~B}-\mathrm{C} 2 \mathrm{~B}-\mathrm{S} 1 \mathrm{~B}$ & $122.34(14)$ \\
\hline $\mathrm{N} 3 \mathrm{~A}-\mathrm{C} 2 \mathrm{~A}-\mathrm{S} 1 \mathrm{~A}$ & $114.70(13)$ & $\mathrm{C} 2 \mathrm{~B}-\mathrm{N} 21 \mathrm{~B}-\mathrm{H} 21 \mathrm{~B}$ & 120.0 \\
\hline $\mathrm{C} 2 \mathrm{~A}-\mathrm{N} 21 \mathrm{~A}-\mathrm{H} 21 \mathrm{~A}$ & 120.0 & $\mathrm{C} 2 \mathrm{~B}-\mathrm{N} 21 \mathrm{~B}-\mathrm{H} 22 \mathrm{~B}$ & 120.0 \\
\hline $\mathrm{C} 2 \mathrm{~A}-\mathrm{N} 21 \mathrm{~A}-\mathrm{H} 22 \mathrm{~A}$ & 120.0 & $\mathrm{H} 21 \mathrm{~B}-\mathrm{N} 21 \mathrm{~B}-\mathrm{H} 22 \mathrm{~B}$ & 120.0 \\
\hline $\mathrm{H} 21 \mathrm{~A}-\mathrm{N} 21 \mathrm{~A}-\mathrm{H} 22 \mathrm{~A}$ & 120.0 & $\mathrm{C} 2 \mathrm{~B}-\mathrm{N} 3 \mathrm{~B}-\mathrm{C} 4 \mathrm{~B}$ & $110.29(16)$ \\
\hline
\end{tabular}




\begin{tabular}{|c|c|c|c|}
\hline $\mathrm{C} 2 \mathrm{~A}-\mathrm{N} 3 \mathrm{~A}-\mathrm{C} 4 \mathrm{~A}$ & $109.72(15)$ & $\mathrm{N} 3 \mathrm{~B}-\mathrm{C} 4 \mathrm{~B}-\mathrm{C} 5 \mathrm{~B}$ & $116.59(16)$ \\
\hline $\mathrm{N} 3 \mathrm{~A}-\mathrm{C} 4 \mathrm{~A}-\mathrm{C} 5 \mathrm{~A}$ & $117.41(16)$ & $\mathrm{N} 3 \mathrm{~B}-\mathrm{C} 4 \mathrm{~B}-\mathrm{C} 41 \mathrm{~B}$ & $116.98(16)$ \\
\hline $\mathrm{N} 3 \mathrm{~A}-\mathrm{C} 4 \mathrm{~A}-\mathrm{C} 41 \mathrm{~A}$ & $117.27(16)$ & $\mathrm{C} 5 \mathrm{~B}-\mathrm{C} 4 \mathrm{~B}-\mathrm{C} 41 \mathrm{~B}$ & $126.28(16)$ \\
\hline $\mathrm{C} 5 \mathrm{~A}-\mathrm{C} 4 \mathrm{~A}-\mathrm{C} 41 \mathrm{~A}$ & $125.31(16)$ & $\mathrm{C} 4 \mathrm{~B}-\mathrm{C} 5 \mathrm{~B}-\mathrm{C} 51 \mathrm{~B}$ & $129.44(17)$ \\
\hline $\mathrm{O} 41 \mathrm{~A}-\mathrm{C} 41 \mathrm{~A}-\mathrm{O} 42 \mathrm{~A}$ & $124.75(16)$ & $\mathrm{C} 4 \mathrm{~B}-\mathrm{C} 5 \mathrm{~B}-\mathrm{S} 1 \mathrm{~B}$ & $109.36(13)$ \\
\hline $\mathrm{O} 41 \mathrm{~A}-\mathrm{C} 41 \mathrm{~A}-\mathrm{C} 4 \mathrm{~A}$ & $124.68(16)$ & $\mathrm{C} 51 \mathrm{~B}-\mathrm{C} 5 \mathrm{~B}-\mathrm{S} 1 \mathrm{~B}$ & $121.04(14)$ \\
\hline $\mathrm{O} 42 \mathrm{~A}-\mathrm{C} 41 \mathrm{~A}-\mathrm{C} 4 \mathrm{~A}$ & $110.53(15)$ & $\mathrm{O} 41 \mathrm{~B}-\mathrm{C} 41 \mathrm{~B}-\mathrm{O} 42 \mathrm{~B}$ & $124.43(17)$ \\
\hline $\mathrm{C} 41 \mathrm{~A}-\mathrm{O} 42 \mathrm{~A}-\mathrm{C} 42 \mathrm{~A}$ & $115.77(14)$ & $\mathrm{O} 41 \mathrm{~B}-\mathrm{C} 41 \mathrm{~B}-\mathrm{C} 4 \mathrm{~B}$ & $124.24(17)$ \\
\hline $\mathrm{O} 42 \mathrm{~A}-\mathrm{C} 42 \mathrm{~A}-\mathrm{C} 43 \mathrm{~A}$ & $106.94(15)$ & $\mathrm{O} 42 \mathrm{~B}-\mathrm{C} 41 \mathrm{~B}-\mathrm{C} 4 \mathrm{~B}$ & $111.25(16)$ \\
\hline $\mathrm{O} 42 \mathrm{~A}-\mathrm{C} 42 \mathrm{~A}-\mathrm{H} 41 \mathrm{~A}$ & 110.3 & $\mathrm{C} 41 \mathrm{~B}-\mathrm{O} 42 \mathrm{~B}-\mathrm{C} 42 \mathrm{~B}$ & $115.04(15)$ \\
\hline $\mathrm{C} 43 \mathrm{~A}-\mathrm{C} 42 \mathrm{~A}-\mathrm{H} 41 \mathrm{~A}$ & 110.3 & $\mathrm{O} 42 \mathrm{~B}-\mathrm{C} 42 \mathrm{~B}-\mathrm{C} 43 \mathrm{~B}$ & $107.72(16)$ \\
\hline $\mathrm{O} 42 \mathrm{~A}-\mathrm{C} 42 \mathrm{~A}-\mathrm{H} 42 \mathrm{~A}$ & 110.3 & $\mathrm{O} 42 \mathrm{~B}-\mathrm{C} 42 \mathrm{~B}-\mathrm{H} 41 \mathrm{~B}$ & 110.2 \\
\hline $\mathrm{C} 43 \mathrm{~A}-\mathrm{C} 42 \mathrm{~A}-\mathrm{H} 42 \mathrm{~A}$ & 110.3 & $\mathrm{C} 43 \mathrm{~B}-\mathrm{C} 42 \mathrm{~B}-\mathrm{H} 41 \mathrm{~B}$ & 110.2 \\
\hline $\mathrm{H} 41 \mathrm{~A}-\mathrm{C} 42 \mathrm{~A}-\mathrm{H} 42 \mathrm{~A}$ & 108.6 & $\mathrm{O} 42 \mathrm{~B}-\mathrm{C} 42 \mathrm{~B}-\mathrm{H} 42 \mathrm{~B}$ & 110.2 \\
\hline $\mathrm{C} 42 \mathrm{~A}-\mathrm{C} 43 \mathrm{~A}-\mathrm{H} 43 \mathrm{~A}$ & 109.5 & $\mathrm{C} 43 \mathrm{~B}-\mathrm{C} 42 \mathrm{~B}-\mathrm{H} 42 \mathrm{~B}$ & 110.2 \\
\hline $\mathrm{C} 42 \mathrm{~A}-\mathrm{C} 43 \mathrm{~A}-\mathrm{H} 44 \mathrm{~A}$ & 109.5 & $\mathrm{H} 41 \mathrm{~B}-\mathrm{C} 42 \mathrm{~B}-\mathrm{H} 42 \mathrm{~B}$ & 108.5 \\
\hline $\mathrm{H} 43 \mathrm{~A}-\mathrm{C} 43 \mathrm{~A}-\mathrm{H} 44 \mathrm{~A}$ & 109.5 & $\mathrm{C} 42 \mathrm{~B}-\mathrm{C} 43 \mathrm{~B}-\mathrm{H} 43 \mathrm{~B}$ & 109.5 \\
\hline $\mathrm{C} 42 \mathrm{~A}-\mathrm{C} 43 \mathrm{~A}-\mathrm{H} 45 \mathrm{~A}$ & 109.5 & $\mathrm{C} 42 \mathrm{~B}-\mathrm{C} 43 \mathrm{~B}-\mathrm{H} 44 \mathrm{~B}$ & 109.5 \\
\hline $\mathrm{H} 43 \mathrm{~A}-\mathrm{C} 43 \mathrm{~A}-\mathrm{H} 45 \mathrm{~A}$ & 109.5 & $\mathrm{H} 43 \mathrm{~B}-\mathrm{C} 43 \mathrm{~B}-\mathrm{H} 44 \mathrm{~B}$ & 109.5 \\
\hline $\mathrm{H} 44 \mathrm{~A}-\mathrm{C} 43 \mathrm{~A}-\mathrm{H} 45 \mathrm{~A}$ & 109.5 & $\mathrm{C} 42 \mathrm{~B}-\mathrm{C} 43 \mathrm{~B}-\mathrm{H} 45 \mathrm{~B}$ & 109.5 \\
\hline $\mathrm{C} 4 \mathrm{~A}-\mathrm{C} 5 \mathrm{~A}-\mathrm{C} 51 \mathrm{~A}$ & $127.36(16)$ & $\mathrm{H} 43 \mathrm{~B}-\mathrm{C} 43 \mathrm{~B}-\mathrm{H} 45 \mathrm{~B}$ & 109.5 \\
\hline $\mathrm{C} 4 \mathrm{~A}-\mathrm{C} 5 \mathrm{~A}-\mathrm{S} 1 \mathrm{~A}$ & $109.35(13)$ & $\mathrm{H} 44 \mathrm{~B}-\mathrm{C} 43 \mathrm{~B}-\mathrm{H} 45 \mathrm{~B}$ & 109.5 \\
\hline $\mathrm{C} 51 \mathrm{~A}-\mathrm{C} 5 \mathrm{~A}-\mathrm{S} 1 \mathrm{~A}$ & $123.15(14)$ & $\mathrm{O} 51 \mathrm{~B}-\mathrm{C} 51 \mathrm{~B}-\mathrm{O} 52 \mathrm{~B}$ & $124.24(17)$ \\
\hline $\mathrm{O} 51 \mathrm{~A}-\mathrm{C} 51 \mathrm{~A}-\mathrm{O} 52 \mathrm{~A}$ & $124.18(17)$ & $\mathrm{O} 51 \mathrm{~B}-\mathrm{C} 51 \mathrm{~B}-\mathrm{C} 5 \mathrm{~B}$ & $124.79(17)$ \\
\hline $\mathrm{O} 51 \mathrm{~A}-\mathrm{C} 51 \mathrm{~A}-\mathrm{C} 5 \mathrm{~A}$ & $123.96(17)$ & $\mathrm{O} 52 \mathrm{~B}-\mathrm{C} 51 \mathrm{~B}-\mathrm{C} 5 \mathrm{~B}$ & $110.94(15)$ \\
\hline $\mathrm{O} 52 \mathrm{~A}-\mathrm{C} 51 \mathrm{~A}-\mathrm{C} 5 \mathrm{~A}$ & $111.85(15)$ & $\mathrm{C} 51 \mathrm{~B}-\mathrm{O} 52 \mathrm{~B}-\mathrm{C} 52 \mathrm{~B}$ & $115.76(14)$ \\
\hline $\mathrm{C} 51 \mathrm{~A}-\mathrm{O} 52 \mathrm{~A}-\mathrm{C} 52 \mathrm{~A}$ & $115.84(14)$ & $\mathrm{O} 52 \mathrm{~B}-\mathrm{C} 52 \mathrm{~B}-\mathrm{C} 53 \mathrm{~B}$ & $107.19(16)$ \\
\hline $\mathrm{O} 52 \mathrm{~A}-\mathrm{C} 52 \mathrm{~A}-\mathrm{C} 53 \mathrm{~A}$ & $106.78(16)$ & $\mathrm{O} 52 \mathrm{~B}-\mathrm{C} 52 \mathrm{~B}-\mathrm{H} 51 \mathrm{~B}$ & 110.3 \\
\hline $\mathrm{O} 52 \mathrm{~A}-\mathrm{C} 52 \mathrm{~A}-\mathrm{H} 51 \mathrm{~A}$ & 110.4 & $\mathrm{C} 53 \mathrm{~B}-\mathrm{C} 52 \mathrm{~B}-\mathrm{H} 51 \mathrm{~B}$ & 110.3 \\
\hline $\mathrm{C} 53 \mathrm{~A}-\mathrm{C} 52 \mathrm{~A}-\mathrm{H} 51 \mathrm{~A}$ & 110.4 & $\mathrm{O} 52 \mathrm{~B}-\mathrm{C} 52 \mathrm{~B}-\mathrm{H} 52 \mathrm{~B}$ & 110.3 \\
\hline $\mathrm{O} 52 \mathrm{~A}-\mathrm{C} 52 \mathrm{~A}-\mathrm{H} 52 \mathrm{~A}$ & 110.4 & $\mathrm{C} 53 \mathrm{~B}-\mathrm{C} 52 \mathrm{~B}-\mathrm{H} 52 \mathrm{~B}$ & 110.3 \\
\hline $\mathrm{C} 53 \mathrm{~A}-\mathrm{C} 52 \mathrm{~A}-\mathrm{H} 52 \mathrm{~A}$ & 110.4 & $\mathrm{H} 51 \mathrm{~B}-\mathrm{C} 52 \mathrm{~B}-\mathrm{H} 52 \mathrm{~B}$ & 108.5 \\
\hline $\mathrm{H} 51 \mathrm{~A}-\mathrm{C} 52 \mathrm{~A}-\mathrm{H} 52 \mathrm{~A}$ & 108.6 & $\mathrm{C} 52 \mathrm{~B}-\mathrm{C} 53 \mathrm{~B}-\mathrm{H} 53 \mathrm{~B}$ & 109.5 \\
\hline $\mathrm{C} 52 \mathrm{~A}-\mathrm{C} 53 \mathrm{~A}-\mathrm{H} 53 \mathrm{~A}$ & 109.5 & $\mathrm{C} 52 \mathrm{~B}-\mathrm{C} 53 \mathrm{~B}-\mathrm{H} 54 \mathrm{~B}$ & 109.5 \\
\hline $\mathrm{C} 52 \mathrm{~A}-\mathrm{C} 53 \mathrm{~A}-\mathrm{H} 54 \mathrm{~A}$ & 109.5 & $\mathrm{H} 53 \mathrm{~B}-\mathrm{C} 53 \mathrm{~B}-\mathrm{H} 54 \mathrm{~B}$ & 109.5 \\
\hline $\mathrm{H} 53 \mathrm{~A}-\mathrm{C} 53 \mathrm{~A}-\mathrm{H} 54 \mathrm{~A}$ & 109.5 & $\mathrm{C} 52 \mathrm{~B}-\mathrm{C} 53 \mathrm{~B}-\mathrm{H} 55 \mathrm{~B}$ & 109.5 \\
\hline $\mathrm{C} 52 \mathrm{~A}-\mathrm{C} 53 \mathrm{~A}-\mathrm{H} 55 \mathrm{~A}$ & 109.5 & $\mathrm{H} 53 \mathrm{~B}-\mathrm{C} 53 \mathrm{~B}-\mathrm{H} 55 \mathrm{~B}$ & 109.5 \\
\hline $\mathrm{H} 53 \mathrm{~A}-\mathrm{C} 53 \mathrm{~A}-\mathrm{H} 55 \mathrm{~A}$ & 109.5 & $\mathrm{H} 54 \mathrm{~B}-\mathrm{C} 53 \mathrm{~B}-\mathrm{H} 55 \mathrm{~B}$ & 109.5 \\
\hline $\mathrm{H} 54 \mathrm{~A}-\mathrm{C} 53 \mathrm{~A}-\mathrm{H} 55 \mathrm{~A}$ & 109.5 & $\mathrm{H} 1 \mathrm{~W}-\mathrm{O} 1 \mathrm{~W}-\mathrm{H} 2 \mathrm{~W}$ & $108(2)$ \\
\hline $\mathrm{C} 2 \mathrm{~B}-\mathrm{S} 1 \mathrm{~B}-\mathrm{C} 5 \mathrm{~B}$ & $88.98(9)$ & & \\
\hline $\mathrm{C} 5 \mathrm{~A}-\mathrm{S} 1 \mathrm{~A}-\mathrm{C} 2 \mathrm{~A}-\mathrm{N} 21 \mathrm{~A}$ & $-179.15(17)$ & $\mathrm{C} 5 \mathrm{~B}-\mathrm{S} 1 \mathrm{~B}-\mathrm{C} 2 \mathrm{~B}-\mathrm{N} 3 \mathrm{~B}$ & $-0.39(16)$ \\
\hline $\mathrm{C} 5 \mathrm{~A}-\mathrm{S} 1 \mathrm{~A}-\mathrm{C} 2 \mathrm{~A}-\mathrm{N} 3 \mathrm{~A}$ & $0.81(15)$ & $\mathrm{C} 5 \mathrm{~B}-\mathrm{S} 1 \mathrm{~B}-\mathrm{C} 2 \mathrm{~B}-\mathrm{N} 21 \mathrm{~B}$ & $179.86(19)$ \\
\hline $\mathrm{N} 21 \mathrm{~A}-\mathrm{C} 2 \mathrm{~A}-\mathrm{N} 3 \mathrm{~A}-\mathrm{C} 4 \mathrm{~A}$ & $179.23(18)$ & $\mathrm{N} 21 \mathrm{~B}-\mathrm{C} 2 \mathrm{~B}-\mathrm{N} 3 \mathrm{~B}-\mathrm{C} 4 \mathrm{~B}$ & $-179.31(19)$ \\
\hline $\mathrm{S} 1 \mathrm{~A}-\mathrm{C} 2 \mathrm{~A}-\mathrm{N} 3 \mathrm{~A}-\mathrm{C} 4 \mathrm{~A}$ & $-0.7(2)$ & $\mathrm{S} 1 \mathrm{~B}-\mathrm{C} 2 \mathrm{~B}-\mathrm{N} 3 \mathrm{~B}-\mathrm{C} 4 \mathrm{~B}$ & $0.9(2)$ \\
\hline $\mathrm{C} 2 \mathrm{~A}-\mathrm{N} 3 \mathrm{~A}-\mathrm{C} 4 \mathrm{~A}-\mathrm{C} 5 \mathrm{~A}$ & $0.2(2)$ & $\mathrm{C} 2 \mathrm{~B}-\mathrm{N} 3 \mathrm{~B}-\mathrm{C} 4 \mathrm{~B}-\mathrm{C} 5 \mathrm{~B}$ & $-1.2(2)$ \\
\hline $\mathrm{C} 2 \mathrm{~A}-\mathrm{N} 3 \mathrm{~A}-\mathrm{C} 4 \mathrm{~A}-\mathrm{C} 41 \mathrm{~A}$ & $-178.60(16)$ & $\mathrm{C} 2 \mathrm{~B}-\mathrm{N} 3 \mathrm{~B}-\mathrm{C} 4 \mathrm{~B}-\mathrm{C} 41 \mathrm{~B}$ & $174.65(17)$ \\
\hline $\mathrm{N} 3 \mathrm{~A}-\mathrm{C} 4 \mathrm{~A}-\mathrm{C} 41 \mathrm{~A}-\mathrm{O} 41 \mathrm{~A}$ & $113.2(2)$ & $\mathrm{N} 3 \mathrm{~B}-\mathrm{C} 4 \mathrm{~B}-\mathrm{C} 5 \mathrm{~B}-\mathrm{C} 51 \mathrm{~B}$ & $-174.50(18)$ \\
\hline
\end{tabular}


